Original Article

\title{
The immediate effects of therapeutic keyboard music playing for finger training in adults undergoing hand rehabilitation
}

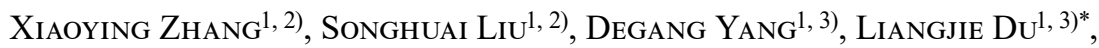 \\ ZIYUAN WANG ${ }^{1,2)}$ \\ 1) Rehabilitation Medicine Faculty, Capital Medical University, China Rehabilitation Research Center: \\ No.10 North Road of Jiaomen, Fengtai District, Beijing 100068, China \\ 2) Department of Psychology, China Rehabilitation Research Center, China \\ 3) Department of Spinal and Neural Function Reconstruction, China Rehabilitation Research Center, \\ China
}

\begin{abstract}
Purpose] The purpose of this study was to examine the immediate effects of therapeutic keyboard music playing on the finger function of subjects' hands through measurements of the joint position error test, surface electromyography, probe reaction time, and writing time. [Subjects and Methods] Ten subjects were divided randomly into experimental and control groups. The experimental group used therapeutic keyboard music playing and the control group used grip training. All subjects were assessed and evaluated by the joint position error test, surface electromyography, probe reaction time, and writing time. [Results] After accomplishing therapeutic keyboard music playing and grip training, surface electromyography of the two groups showed no significant change, but joint position error test, probe reaction time, and writing time obviously improved. [Conclusion] These results suggest that therapeutic keyboard music playing is an effective and novel treatment for improving joint position error test scores, probe reaction time, and writing time, and it should be promoted widely in clinics.

Key words: Therapeutic Keyboard Music Playing (TKMP), Finger training, Music therapy
\end{abstract}

(This article was submitted Feb. 24, 2016, and was accepted May 14, 2016)

\section{INTRODUCTION}

The therapeutic instrument music performance (TIMP) technique uses instruments to reinforce functional motor patterns; the instruments are usually percussive instruments, which do not require any previous music skills ${ }^{1)}$. Instrumental piano training entails the repetition of fine finger movements as well as finger and hand coordination. Furthermore, those performing the movement receive immediate auditory feedback regarding performance and can therefore try to correct their own performance ${ }^{2}$. For fine motor skill training, TIMP usually uses the keyboard as its main rehabilitative intervention instrument. Therapeutic keyboard music playing (TKMP) uses familiar melodies of famous music pieces to explore a new type of TIMP.

According to past research, TKMP not only provides pleasure from making audible music but also motivates individuals to participate in rehabilitation by using their affected and intact hand functions ${ }^{1}$. Although there has been some research supporting the application of TIMP for brain injury, cerebral palsy, and neuro-rehabilitation, less research supports TKMP used for physical therapy (PT). Actually, TIMP, especially the TKMP method, is very suitable for patients with hand dysfunctions.

Rehabilitation of finger movement function during PT for patients with spinal cord injuries is based on more precise controlling exercises. The PT exercises were designed in the form of challenging games. Patients were asked to perform to

\footnotetext{
*Corresponding author. Liangjie Du (E-mail: Strongneck@qq.com)

(C)2016 The Society of Physical Therapy Science. Published by IPEC Inc.

This is an open-access article distributed under the terms of the Creative Commons Attribution Non-Commercial No Derivatives (by-nc-nd) License $<$ http://creativecommons.org/licenses/by-nc-nd/4.0/>.
} 
the best of their ability, but the patients always felt tired. However, TKMP is a new way to facilitate functional non-musical outcomes by training and retraining the hand and fingers to achieve motor control and hand muscle recovery.

This experiment was used to observe the immediate effects of TKMP on variations in the joint position error (JPE) test, surface electromyography (sEMG), probe reaction time (P-RT), and writing time after intervention. In addition, it was also performed to explore a more effective method that includes physical hand exercises and TKMP techniques to help in the recovery of patients with hand dysfunction.

\section{SUBJECTS AND METHODS}

Ten young adults who needed finger training were selected. The subjects had a mean age of $26.4 \pm 3.1$ years, an average height of $170.0 \pm 5.6 \mathrm{~cm}$, and a mean body weight of $64.5 \pm 7.2 \mathrm{~kg}$. They were selected at random from the medical school interns who were able to independently perform activities of daily living. They participated in the experimental group and in the control group on different days. The experimental group was administered the TKMP therapy. The same subjects were also used as the control group and performed grip training, but not on the same days as when TKMP therapy was performed. All subject characteristics are detailed in Table 1.

Before the experiment, all subjects were tested using the following: JPE test, sEMG, P-RT, and writing time. After all interventions were accomplished, subjects were evaluated by using the tests again to observe the intervention effects. The purpose and contents were explained to the subjects before the experiment, and they gave their informed consent to participate. All experimental procedures in this study were explained to them and were approved by the Research Ethics Committee of the China Rehabilitation Research Center (IRB no. 2015-zx-09).

The subjects sat on low chairs with their shoulders and backs straight, and they placed their right hand naturally on the keyboard. In the experimental group, the subjects were trained to play musical pieces for approximately 20 minutes under the guidance of the music therapist. The music piece "Ode to Joy," which was written by the famous German classical composer Beethoven, was familiar to all the experimental group participants. The melody used in this experiment was only eight bars with 42 beats in $\mathrm{C}$ major. In the control group, the subjects were trained with grip training under the guidance of the physical therapist. Then, all subjects were assessed and evaluated by the tests previously mentioned.

The JPE test is considered the primary measure of neck proprioception ${ }^{3}$. It is also considered the primary measure of upper limb proprioception and has been widely used as an outcome measure for patients with cervical spinal cord injury4). Although there are few reports to support its use for measuring other joints, it has been widely used as an effective evaluation of angle joints. During the JPE test, ROM measurement equipment (BioVal 4.51, SyCoMoRe 8.51, RMIngenierie, France) was used. The fixed and mobile arms were the vertical axis of the radius and fifth metacarpal; the extension angles of the wrist joints were recorded by the computer. The subjects were asked to close their eyes, and the examiner extended the subjects' wrist joints at random angles. The subjects' wrist joints were put back on tables, and they were asked to extend their wrists at approximately the same angles as in the initial extension. Errors in wrist extension angles were recorded. Each measurement was performed five times, and the average value was used for analysis.

The muscle strength power test was performed using sEMG. sEMG tests the muscle function through the inquiry of the electrical signal that the muscle emanates ${ }^{5}$. It is reported that $\mathrm{sEMG}$ evaluation data are usually collected by an sEMG system (Telemyo 2400T; Noraxon, Scottsdale, AZ, USA) ${ }^{6}$. In this experiment, sEMG showed the peak value and average of muscle strength power. The data were tested both before and after the intervention.

The P-RT was tested using a digital audio player/recorder (RIO Japan). The recorder was used to play the stimulus signals to the subject and the subject's vocal response was recorded through the microphone. A digital audio player/recorder was used to record the vocal response that the subject had in response to the stimulus signal. The sounds "yoi" and "pi" were pre-recorded on the RIO player. "Yoi" gave notice that the test would begin, and "pi" was the stimulus signal. The subjects were required to respond to the auditory cue "pi" by loudly saying "pa" as quickly as possible. The data were processed using Digion-Sound 5 (Digion) software. The results were downloaded to a PC, and the time between the auditory stimulus of "pi" and the utterance of "pa" during P-RT was evaluated").

During the writing time evaluation, the subjects were asked to write the uppercase English alphabet with their left hand on A4 paper. There were writing grids with $1-\mathrm{cm}^{2}$ areas on the A4 paper. The time it took to write the uppercase English alphabet was measured.

All the subjects in the experimental group were trained with the same musical pieces and the same playing method with the five fingers of their right hand. During the 20-minute therapeutic sessions, the music therapist first taught them the single melody; when the subjects were accustomed to playing the melody, the music therapist began to accompany them with the same harmony until they were quite skilled in playing. The musical pieces played by the experimental group with their right hands are shown in Fig. 1.

Two-way repeated-measures analysis of variance (ANOVA) was used to assess and evaluate statistically significant differences in the intervention of the two groups. If any significant interaction was found, then the paired t-test was used to compare the outcome indicators before and after the intervention. Data were analyzed with SPSS version 17.0 for Windows (SPSS, Chicago, IL, USA). The level of statistical significance was set at 0.05 . 
Table 1. Subject characteristics

\begin{tabular}{lc}
\hline & Mean \pm SD \\
& $\mathrm{N}=10$ \\
\hline Age $(\mathrm{yrs})$ & $26.4 \pm 3.1$ \\
Height $(\mathrm{cm})$ & $170.0 \pm 5.6$ \\
Weight $(\mathrm{kg})$ & $64.5 \pm 7.2$ \\
\hline
\end{tabular}

Ode to Joy

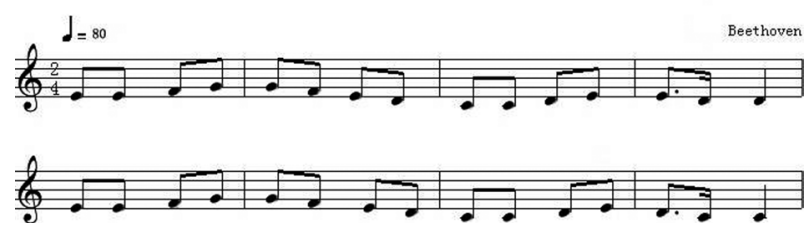

Fig. 1. Musical piece played by the experimental group with their right hands

Table 2. Intervention effects of TMKP

\begin{tabular}{lccccl}
\hline & $\begin{array}{c}\text { a. Experiment group, } \\
\text { before }\end{array}$ & $\begin{array}{c}\text { b. Experiment group, } \\
\text { after }\end{array}$ & $\begin{array}{c}\text { c. Control group, } \\
\text { before }\end{array}$ & $\begin{array}{c}\text { d. Control group, } \\
\text { after }\end{array}$ & \\
\hline JPS $\left(^{\circ}\right)$ & $5.4 \pm 1.9$ & $3.2 \pm 1.2$ & $3.6 \pm 1.6$ & $3.4 \pm 1.6$ & $\mathrm{~b}<\mathrm{a}, \mathrm{c}, \mathrm{d} *$ \\
Ave sEMG $(\mu \mathrm{V})$ & $951.4 \pm 914.9$ & $839.2 \pm 496.6$ & $1,061.4 \pm 635.2$ & $890.3 \pm 505.2$ & \\
Max sEMG $(\mu \mathrm{V})$ & $4,827.7 \pm 3,951.3$ & $5,347.2 \pm 3,086.5$ & $5,996.7 \pm 4,357.0$ & $6,061.0 \pm 3,123.4$ & \\
P-RT $(\mathrm{ms})$ & $383.2 \pm 81.5$ & $323.7 \pm 53.4$ & $399.3 \pm 112.0$ & $426.8 \pm 155.8$ & $\mathrm{~b}<\mathrm{a}, \mathrm{c}, \mathrm{d} *$ \\
Writing Time $(\mathrm{s})$ & $21.9 \pm 3.4$ & $18.8 \pm 4.4$ & $21.7 \pm 4.0$ & $21.6 \pm 3.6$ & $\mathrm{~b}<\mathrm{a}, \mathrm{c}, \mathrm{d} *$ \\
\hline
\end{tabular}

$* \mathrm{p}<0.05 ; \mathrm{p}<0.01$

\section{RESULTS}

The data for the JPE test, sEMG, P-RT, and writing time for the two groups are shown in Table 2.

There were no significant differences in average and maximum muscle strength; however, there was an obvious interactive function of the JPE test and P-RT in the two groups. The paired t-test results after TMKP showed that writing time, P-RT, and JPE test score all significantly decreased. The control group showed no significant differences.

\section{DISCUSSION}

TKMP was used as an intervening variable to train the experimental group subjects to improve their JPE test, sEMG, P-RT, and writing time results. Grip training was used to train the control group subjects so that the results could be compared to those of the experimental group trained with the TKMP method. Results are shown in Table 2. There were no significant differences in the maximum sEMG between the two groups, which meant that grip training played the same role as TKMP training for muscle strength. The experimental group subjects reported feeling joyful and interested during the music training, but there was no obvious enhancement on sEMG.

According to the data shown in Table 2, there was a significant improvement in JPE test, P-RT, and writing time. This meant that the TKMP intervention was more effective than grip training for reinforcing finger movement functions. Compared to other traditional therapy methods such as grip training or other occupational therapy, the TKMP method provided tremendous advantages. In this experiment, the famous music work "Ode to Joy," which was very familiar to every subject, was used. It was easy for the subjects to follow the melody and learn how to play. The melody, which was easy for the subjects to memorize, played an active role in the session. Although the active psychological effects of the music were not evaluated during this experiment, they were evident in the writing time and P-RT results. Music, as an acoustic expression of art, has unique aesthetic functions that could help improve the training efficacy. In addition, TKMP combined with a familiar melody provided an interesting way to practice hand muscle function and was more diverse and effective than traditional occupational therapy or PT. It was motivating and engaging for the subjects. Finally, for writing time, there was an obvious enhancement in speed after keyboard playing that was not seen after grip training. Therefore, TKMP is an effective and novel treatment that should be widely promoted in clinics.

\section{REFERENCES}

1) Chong HJ, Cho SR, Jeong E, et al.: Finger exercise with keyboard playing in adults with cerebral palsy: a preliminary study. J Exerc Rehabil, 2013 , 9: 420-425. [Medline] [CrossRef]

2) Lampe R, Thienel A, Mitternacht J, et al.: Piano training in youths with hand motor impairments after damage to the developing brain. Neuropsychiatr Dis 
Treat, 2015, 11: 1929-1938. [Medline] [CrossRef]

3) Zheng T, Huo M, Maruyama H, et al.: Effects on ROM and joint position sense of the neck of two different interventions. J Phys Ther Sci, 2015, 27: 1041-1043. [Medline] [CrossRef]

4) Chen X, Treleaven J: The effect of neck torsion on joint position error in subjects with chronic neck pain. Man Ther, 2013, 18: 562-567. [Medline] [CrossRef]

5) Criswell E: Cram's introduction to surface Electromyography. Jones and Bartlett, 2011, 3.

6) Huang Q, Wang KY, Yu L, et al.: Evaluation of the effects of different treatments for the elbow joint using joint proprioception and surface electromyography. J Phys Ther Sci, 2015, 27: 3907-3909. [Medline] [CrossRef]

7) Huang Q, Maruyama H, Huo M: Probe reaction time changes with fatigue induced by climbing up-and-down stairs. J Phys Ther Sci, 2012 , 24: 995-997. [CrossRef] 